\title{
The Distributed Photovoltaic Demand Response Model Based on Security and Economy in Active Distribution Network
}

\author{
Li Lin ${ }^{1,2}$, Cao Jun², Tao Weiqing ${ }^{1}$ \\ ${ }^{1}$ School of Electric Engineering and Automation, Hefei University of Technology, Hefei, China \\ ${ }^{2}$ CSG Smart Grid Electrical Technology CO., Ltd., Hefei, China
}

Email address:

lilin@csg.com.cn (Li Lin), caojun407@126.com (Cao Jun)

\section{To cite this article:}

Li Lin, Cao Jun, Tao Weiqing. The Distributed Photovoltaic Demand Response Model Based on Security and Economy in Active Distribution Network. Automation, Control and Intelligent Systems. Vol. 4, No. 3, 2016, pp. 53-58. doi: 10.11648/j.acis.20160403.11

Received: April 26, 2016; Accepted: June 6, 2016; Published: June 8, 2016

\begin{abstract}
The technology of demand side response introduced in distributed photovoltaic power supply was proposed in this paper. Analyzing the applicability of demand response which established by distributed photovoltaic, presenting system implementation architecture and basic technical requirements. Without considering the input of energy storage equipment, establishing the demand response model in the active distributed network based on combination of power prediction, load forecasting and weather mode prediction. The technical characteristics of system power, load and environment was analyzed, and the security and economy of distributed generation integration was followed by analyzed. The model will improve the performance of renewable energy operation and consumption, the reliable and economic access capability of large-scale distributed photovoltaic system in the active distribution network.
\end{abstract}

Keywords: Distributed Photovoltaic, Demand Response, Model, Security and Economy

\section{Introduction}

With the recent aggravation of environmental pollution, energy conservation and sustainable energy development faces great opportunities. Renewable energy has become the best choice. High permeability distributed power access has changed the traditional operation mode of the distribution network. The development tendency of the grid is to "active distribution network" [1-2]. The renewable generation resources attitudes of distribution network are changed from past passive restrictions to active guidance and use [3]. Compared with large centralized power generation [4], distributed power has been rapidly developed because of benefits which include reduced the distance between customer and load, increased flexibility and reduced investment costs.

Despite the numerous benefits, there are several challenges faced by Distributed Power Grid security access because of its intermittent and fluctuation features [5]. This is also currently the focus of research literature. Literature [6-7] proposed a containing distributed power supply distribution network fault analysis simulation model and positioning strategy from the point of distribution feeder fault analysis and positioning. The literature [8] studied the branch feeder protection of the distributed power from the perspective of line protection. The literature [9-10] discussed the effect of distribution static voltage network Stability and voltage reactive power control when distributed power access. The literature [11-13] have researched from the perspective of the distribution network access distributed power capacity, analyzed the distributed power accessible capacity under harmonic constraints and load balancing distribution conditions, or the capacity limit under extreme conditions.

From the above literature review, we could find that current technology research focus is mainly consider how to safely and effectively access the distributed power from grid side, and then to propose solutions to the problem may be caused after the access. But there are two aspects problems of these methods. One side, deal with the problem by focusing on how to solve the problems appear later, rather than how to prevention upfront by technical characteristics of distributed 
power. The other side, power grid plan did not fully consider distributed power supply participating in, that lack of interaction, so appear the situation that one side the maximum planned power generation output, the other side the distributed power supply is also by the maximum power output. It is a waste of resources and also a risk of the power grid, the security and economy of the distributed power are not well reflected. Although the economic efficiency can improved by using the energy storage device in the distributed power system, but it is generally used in large centralized power generation and micro-grid pilot, consider of the current price and service life of the energy storage device, it is not economic and realistic by applying to the user-side smaller distributed power.

With the development of demand response technology [14-15], the power users can actively participate in energy-use planning [16], and also improve their energy efficiency enthusiasm. The literature [17-18] pointed out that demand response is an active distribution network technology, it reflects the initiative of the active distribution network. The literature [19-20] focuses on analyzing the necessity of realize the demand side response technology in future new power system. This paper combined the technology trends of active distribution network, taking into consideration photovoltaic power generation (PV) as main style of the current distributed power applications, introduces distributed power demand response technology in PV. By combining the features of distributed photovoltaic capacity is not large, position flexible, and also without considering the poor economy of energy storage device, this paper further proposed demand response model under the active distribution network. The model implements real-time interaction between distributed PV systems and demand side management system to achieve a balance between supply and demand. It is also avoiding the waste of energy, improving distributed photovoltaic grid security and economy, and guaranteeing a reliable consumption of high permeability distributed power in the active distribution network.

\section{Distributed PV Demand Response System Analysis}

\subsection{Distributed PV Applicability Analysis}

Active distribution network as a frontier branch of the smart grid, its internal distribution network mainly be composed by highly permeable distributed power, and the distributed power has two-way flow. The distributed power mainly types has solar power, wind power and micro-gas turbine power and so on. These various types of features are described below:

$\mathrm{PV}$ as the most suitable promotion for renewable energy power generation forms, it has been used widely in European countries. Follow "Spontaneous self-use, the balance online, the power grid adjust" principles, china made clear that it adjust participation function in the power grid. Compared to other forms of distributed generation, PV has features of not high installation conditions and a wide range of capacity, making it very suitable for the industrial, commercial and residential power users, so it is the main body of the distributed power at present.

Wind power is the main form of renewable energy in recent years, because of its power strict restrictions on the installation location, it is not suitable for the urban environment or the lack of wind resource areas, only some remote areas, islands users have strong applicability.

Micro-turbine generation has the multi-fuel, low fuel consumption and high controllability features, it is suitable for urban and remote rural areas, but because of its high fuel reserves requirements, its applicability is weak compared to the previous renewable energy sources.

From the foregoing, different types of distributed power can be used as a user-side resource to participate in the distribution network of interactive response, and PV is considered to be the dominant species of distributed power supply. PV grid-connected is realized by the power electronic devices, and technically provide protection for participating in power grid power or reactive voltage adjustment, without additional investment compensation device. At the same time, the existing distributed power supply is mainly photovoltaic. But $\mathrm{PV}$ is affected by weather conditions, and the system was not configured by any energy storage device, so, intermittent and volatility in the photovoltaic process a significant impact on the safety and stability of the grid. If there is no effective control and regulation technology, after the formation of large-scale, it will seriously endanger the safe operation of the original grid.

\subsection{Containing Distributed Photovoltaic Intelligent Management Unit Demand Response System}

To solve the distributed PV scale access and consumption in active distribution network, and achieve security and economy, demand response mechanism can be introduced in the distributed photovoltaic to coordinate source, network and load operation. Response mechanism of the distributed PV demand side is achieved by intelligent management unit which installed in each system. Figure 1 shows the system structure, distributed intelligent management unit is responsible for the real-time status acquisition, monitoring and controlling of the various components in the system. According to number, size, scope of the system components nodes and the type of information, communication forms can chose fiber, low-voltage power line carrier, wireless Ethernet or other means of communication. According to real-time data state within the system and demand side response strategy, intelligent units generate real-time response information. Demand side management system is installed in the master system side, collecting and analyzing the current response information, combining with the current power grid operation to release information, realize real-time interaction with various distributed photovoltaic equipment, and achieve management control of distributed photovoltaic output. Protecting the current distribution network safe operation when distributed PV in maximize output, and achieve security and economy. 


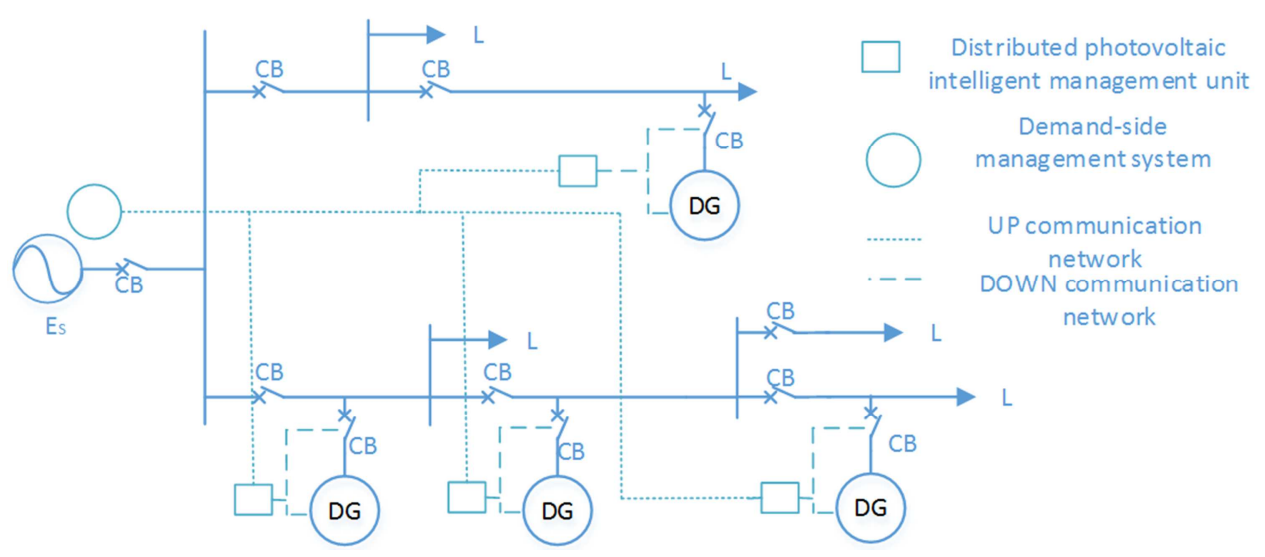

Figure 1. Containing distributed photovoltaic intelligent management unit the demand response system.

\section{Distributed PV Secure and Economic Response Model}

\subsection{Distributed PV Demand Side Response Achieve Foundation}

Demand side response technology guide the user to use less electricity in peak and more electricity in valley by policy setting and economic incentives, which can improve energy efficiency, optimize the power consumption, ease the shortage pressure, low the cost of electricity. Unlike the inherent power consumption mode, its essence is changing the traditional grid user role from passive to active. Power users actively participate in making the variety of electrical safety economic policy of grid demand side, to achieve win-win results. The literature [21] researched the demand response technology implementation in smart home technology application. The literature [22] proposed the demand response technology implementation methods in micro power grid. The literature [23] introduced demand side response technology in electric vehicle orderly charging. Each results of literature show that application of demand response technology enhanced the utilization of corresponding electrical energy system, so the introduction of distributed photovoltaic demand response technology is a viable technical solution. To achieve this technology, the construction of distributed grid PV system needs to have certain technical foundation, the corresponding function are described in Table 1:

Table 1. Distributed PV demand response system technical foundation.

\begin{tabular}{ll}
\hline Infrastructure & Function \\
\hline $\begin{array}{l}\text { Distributed Intelligent } \\
\text { management unit }\end{array}$ & $\begin{array}{l}\text { Distributed PV energy management unit, is responsible for the real-time status monitoring and controlling of the various } \\
\text { components in the system, has the ability to communicate with the lower layer various components, or the upper layer demand } \\
\text { side management system of the master. } \\
\text { Data transmission network of two-way communication between the various components within the distributed photovoltaic and } \\
\text { distributed intelligent management unit, the communication network can be composed of optical fibre, power line carrier, } \\
\text { Ethernet or wireless, etc. }\end{array}$ \\
$\begin{array}{l}\text { Communication network } \\
\text { Analysis }\end{array}$ & $\begin{array}{l}\text { Achievement of the distributed PV field operations data storage and analyze, is the basis of the specific demand side response. } \\
\text { Memoter system can achieve real-time remote control of the each distributed PV key components through communications } \\
\text { network. } \\
\text { Achievement of the stable safety margin of power distribution system and the release of real-time information of the distributed } \\
\text { photovoltaic current operating mode. }\end{array}$ \\
\hline
\end{tabular}

\subsection{Distributed PV Demand Side Response Model}

Demand response technology model is currently established more according to the price incentives. The literature [24] established client TOU(Time Of Use) response model by the demand price which elasticity analysis user power consumption change follow the change of price. The literature [25] established the demand response model by simulated the behavior characteristic of power users when use the TOU. The literature [26] researched the behavior of demand side from micro power grid optimization market economy, established the demand response model and developed simulation evaluation.
This paper, the distributed photovoltaic demand response model established from the viewpoint of grid economic planning, focused on the interaction between both ends, and achieved a balance between supply and demand without considering the source storage equipment investment. At the same time, the volatility and intermittent of distributed $\mathrm{PV}$ is mainly caused by weather conditions, different weather conditions has different output, so distributed PV demand response model need to consider weather model prediction. Compared to the current grid distributed PV which according to the maximum power output, the power output of distributed PV which have achieved demand response technology is dynamically regulated, the 
demand response signal for grid is an analysis result by integrated multiple parameters which including grid demand side management publication, distributed PV power prediction and load forecasting, weather model. Distributed PV demand side response implementation is shown in Figure 2:

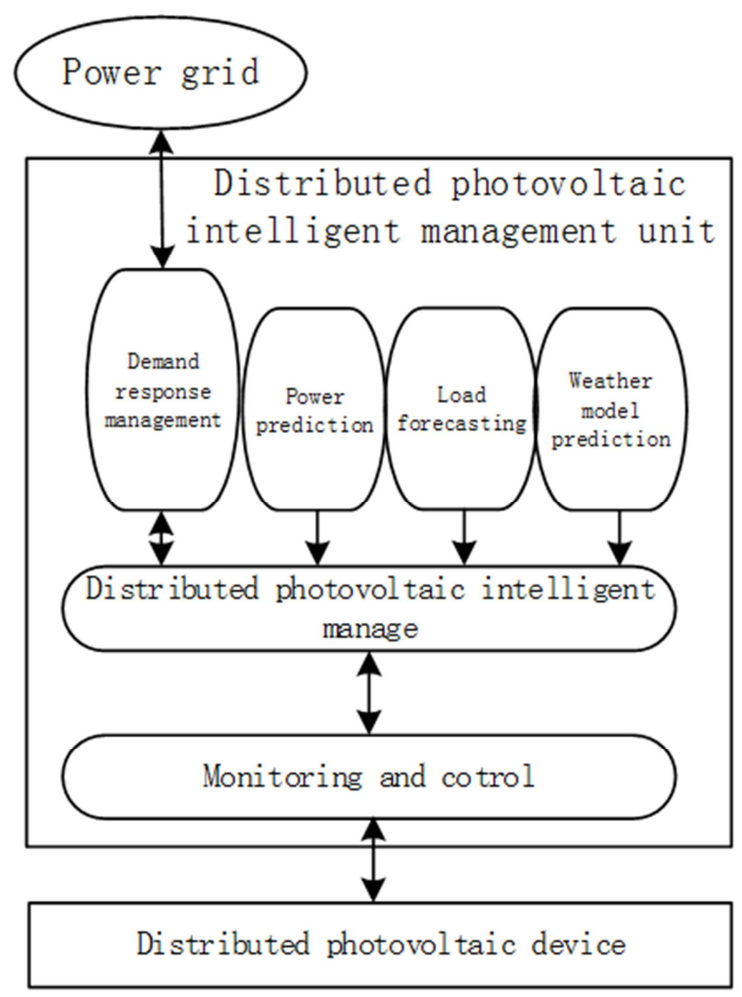

Figure 2. Distributed PV demand side response implementation.

Distributed PV intelligent management unit measures and monitors the various intelligence components of the system, to acquire the real-time status of each component by communication network. Implementation of power prediction, load forecasting and weather model prediction requires the system power output, load nature and environmental characteristics meet the technical requirements, the specific features are described as follows:

(1) Power Output

Distribution network topology including distributed PV is not the radial, the active/reactive power levels and direction is different in access point, therefore the direction of energy flow no longer is from the root node to the load end along the distribution line. Power flow of distributed PV at the access point has the following 3 kinds (DPP: distributed PV active power output, DPL: distributed PV load power):

1) DPP < DPL: The distribution network provide active power at the access point, active trend direction has not changed.

2) $\mathrm{DPP}=\mathrm{DPL}$ : The distribution network provide active power output is 0 at the access point, so all access point load consumption of power provided by the distributed PV.

3) DPP > DPL: The distributed PV reverse injection of active power to distribution network at access point, active trend direction contrary to the original.

\section{(2) Load Nature}

Difference from the fixed load of the traditional distribution network, the load under the active power distribution network is no longer a single species, some of which can response mechanism to adjust the grid, actively participate in grid scheduling. Based on different levels of participation grid scheduling, load will be divided into uncontrollable load, controllable load and adjustable load three categories.

Uncontrollable load: traditional load, whose features is that electricity demand is relatively fixed, is the main part of the current distribution network load.

Controllable Load: also called interruptible load, its realization usually by sign a contract or agreement with electric power company, when the system peak or emergency it can be interrupted or reduced, it is an important guarantee to achieve demand response.

Adjustable Load: that can't fully respond to power grid scheduling. To some extent, it can be able to follow the time-price ladder and other boot mechanisms to regulate its electricity needs, participate in demand response.

(3) Environmental Characteristics

Because the output of distributed PV is affected by weather conditions, so this paper combined the impact of weather on distributed renewable energy output, defined three weather models: good, normal and bad. In good mode, get the optimal utilization of renewable energy, in the normal mode, the utilization of renewable energy is medium, in the bad mode, the utilization of renewable energy is very small. Distributed power can switch in the three modes intelligently, switch between various states transition is shown in Figure 3:

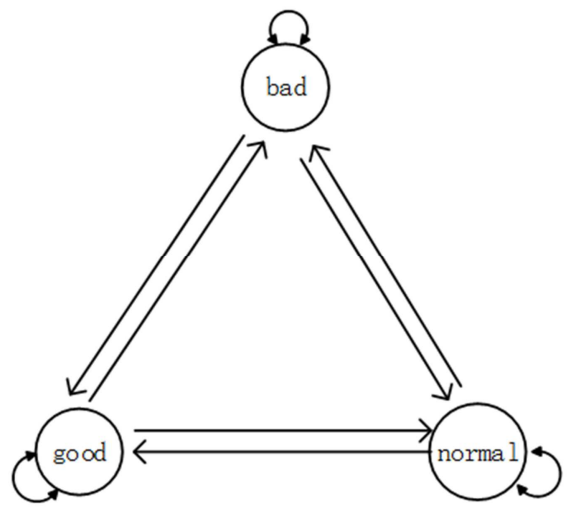

Figure 3. Three models of weather conditions.

\subsection{Distributed PV Secure and Economic Demand Response Achieve}

The distributed PV secure and economic demand response is achieved through two-way communication between the master demand management system and the distributed intelligent management unit. Demand management is responsible for the management and regulation of overall distribution lines according to the power prediction and load forecasting of the unit, timing or real-time release relevant secure and economic policy, ensure system safety and economical. The implementation model is shown in Figure 4: 


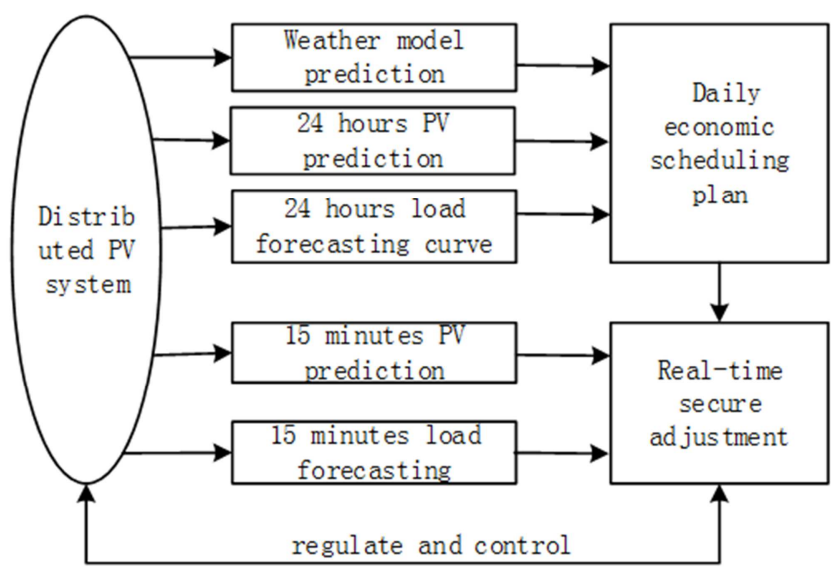

Figure 4. Distributed PV secure and economic implementation model.

Distributed photovoltaic intelligent management unit can obtain the relevant demand side management published strategies, response the corresponding strategies, and manage the various components of subsystem. The core work of distributed photovoltaic intelligent management unit is to realize distributed photovoltaic power prediction and load forecasting, according to a predetermined time (day and 15 minutes) obtain power output curve in the short term, participant demand response, reduce volatility and randomness of distributed photovoltaic. Power prediction algorithm include: neural network algorithm [27], support vector machine algorithm [28], based on Markov chain algorithm [29], and so on.

At the same time, power prediction need to use an weather model estimate its influence, the weather model prediction can use Monte Carlo simulation algorithm [30], to assess the following weather state, improve the accuracy of prediction of the distributed PV output.

To achieve a flexible demand response, load forecasting use month and year for the long-term forecasting, also use day and 15 minutes ultra-short-term or short-term load forecasting. Forecasting methods use prediction algorithm based on time series and the index smoothing algorithm [31].

Based on the above technology, the distributed PV demand response security and economy reflect as follows:

(1) Security reflect

The security of model realized through short time (15 minutes) power load forecasting, monitor and predict by every 15 minutes to solve intermittent and volatility characteristics of distributed photovoltaic. It is minimize the fault solution time. Specific Security content includes line safety and load safety, as follows:

Line safety: Compared with traditional distributed PV maximum power operating mode, using demand response technology make power output is controllable. Through the intelligent management unit, the trend of nodes is subject to monitored by demand side management system, avoiding that the changed trend impact on corresponding feeder protection devices, and causing refusing, misoperation and other serious security hidden danger.

Load safety: Achieved important load priority power supply protection. When the grid load is too large or failure, regulating common load or scheduling nearby distributed PV output, maximize guarantee power supply security of the important load.

(2) Economy reflect

It different from which traditional power generation output is according to the year plan and unilateral largest planning delivery, the economic model is realized by daily power output and load forecasting. Demand side management intelligent scheduling each distributed PV output to achieve the economic by anticipating the next day's power output and load consumption. The economic is reflected as follows:

1) Grid corporation plan a reasonable daily power generation strategy according to the load consumption and distributed PV output forecasting techniques, avoid power invest redundant caused by demand imbalance, save fuel and energy storage equipment investment, and reduce line losses.

2) Make the best of renewable energy, provide power support for the load according to nearby principle, reduce transmission costs and improve low-carbon economy.

3) Improve the capability of the distributed PV access the distribution network, adapt to the development of large-scale distributed PV, delay the investment for the modification of the original power grid capacity because of the economic development and load increases.

At the same time, because of the distributed PV systems not consider the energy storage device, increasing the flexibility and practicality of distributed PV applications, reducing the investment and maintenance costs, further improving the economy.

\section{Conclusion}

Active distribution network has made full attention and rapid development as the direction of the power grid for absorption large-scale distributed power in recent years, related supporting technology is gradually improving. At the same time, demand side response technology changed the traditional electric power consumption mode, achieved the electricity market users to actively participate in power management. It plays an important role in raising electricity utilization efficiency, optimizing electricity using, alleviating the power shortage pressure and reducing power cost. This paper introduced demand side response technology into distributed PV system, combined with power prediction, load forecasting and weather model, proposed the security and economy of distributed photovoltaic demand side response model. It proposed a feasible technical solutions for the active power distribution network acceptance of large-scale distributed PV. However, from the current relevant literature and technological achievements, we can see that the active distribution grid technologies and demand side response technologies are at the exploratory study stage, and not mature, key technologies of the model such as related algorithm accuracy and evaluation need to depth research. 


\section{References}

[1] Fan Mingtian, Zhang Zuping, Su Aoxue, et al. Enabling technologies for active distribution systems [J]. Proccedings of the CSEE, 2013, 33(22): 12-18.

[2] Wang Chengshan, Sun Chongbo, Li Peng. Review and perspective on the optimization of active distribution network [J]. Electric Power Construction, 2015, 36(1): 8-15.

[3] Liu Rui, Yang Jingfei, Cheng Haozhong, et al. Comprehensive evaluation of grid-connected distributed generation [J]. Proceedings of the CSU-EPSA, 2013, 25(1): 34-39.

[4] Ding Ming, Wang Weisheng, Wang Xiuli, et al. A review on the effect of large-scale PV generation on powersystems [J]. Proceedings of the CSEE, 2014, 34(1): 1-14.

[5] Yu Jiancheng, Chi Fujian, Xu Ke. Analysis of the impact of distributed generation on power grid [J]. Proceedings of the CSU-EPSA, 2012, 24(1): 138-141.

[6] Zhao Yonghua, Fang Yongyi, Wang Na, et al. Research on the impacts on feeder automation by inverter-based distribution generation connected to the distribution network [J]. Power System Protection and Control, 2013, 41(24): 117-122.

[7] Liu Jian, Zhang Xiaoqing, Tong Xiangqian, et al. Fault location for distribution systems with distributed generations [J]. Automation of Electric Power Systems, 2013, 37(2): 36-42.

[8] Wang Fei, Duan Jiandong, Liu Wuji, et al. Protection for distribution network lines connected with multi distributed generations containing wind farm $[\mathrm{J}]$. Power System Protection and Control, 2013, 41(10): 68-73.

[9] Zhang Qian, Liao Qingfen, Tang Fei, et al. Steady state voltage stability assessment method of distribution network considering inter-connection of distributed generators [J]. Automation of Electric Power Systems, 2015, 39(15): 42-48.

[10] Wang Xuqiang, Liu Guangyi, Zeng Yuan, et al. Analysis on the effects of Volt/Var control method considering distributed generation [J]. Power System Protection and Control, 2014, 42(1): 47-53.

[11] Zhong Qing, Gao Xinhua, Yu Nanhua, et al. Accommodating capacity and mode of distributed generation under harmonic constraint in active distribution networks [J]. Automation of Electric Power Systems, 2014, 38(24): 108-113.

[12] Liu Jian, Huang Wei. Analysis on grid-connectible capacity of distributed PV generation in case of PV generation distribution close to load distribution [J]. Power System Technology, 2015, 39(2): 299-306.

[13] Huang Wei, Liu Jian, Wei Haokun, et al. Extreme capacity limitations of photovoltaic generators in distribution grids [J]. Power System Protection and Control, 2015, 43(3): 22-28.

[14] Anand M P, Ongsakul W, Singh J G, et al. Impact of economic dispatch in a smart distribution network considering demand response and power market[C]// Energy Economics and Environment (ICEEE), 2015 International Conference on. IEEE, 2015: 1-6.

[15] Soroudi A, Siano P, Keane A. Optimal DR and ESS Scheduling for Distribution Losses Payments Minimization Under Electricity Price Uncertainty[J]. IEEE Transactions on Smart Grid, 2015.
[16] Zhao Huiying, Liu Guangyi, Jia Hongjie, et al. Analysis of demand response program based on refined models [J]. Power System Protection and Control, 2014, 42(1): 62-68.

[17] Liu Guangyi, Zhang Kai, Shu Bin. Six actives and key technologies of active distribution network [J]. Electric Power Construction, 2015, 36(1): 33-37.

[18] Zeng Ming, Han Xu, Li Bo. Study of demand response safeguard mechanism for active $[\mathrm{J}]$. Electric Power Construction, 2015, 36(1): 110-114.

[19] Zeng Bo, Yang Yongqi, Duan Jinhui, et al. Key issues and research prospects for demand-side response in alternate electrical power systems with renewable energy sources $[\mathrm{J}]$. Automation of Electric Power System, 2015, 39(17): 10-18.

[20] Wang Xifan, Xiao Yunpeng, Wang Xiuli. Study and analysis on supply-demand interaction of power system under new circumstances $[\mathrm{J}]$. Proceedings of the CSEE, 2014, 34(29): 5018-5028.

[21] Wang Z, Gu C, Li F, et al. Active Demand Response Using Shared Energy Storage for Household Energy Management [J]. Smart Grid IEEE Transactions on, 2013, 4(4): 1888-1897.

[22] Zhu Lan, Yan Zheng, Yang Xiu, et al. Integrated resources planning in microgrid based on modeling demand response [J]. Proceedings of the CSEE, 2014, 34(16): 2621-2628.

[23] Ge Shaoyun, Guo Jianyi, Liu Hong, et al. Impacts of electric vehicle's ordered charging on power grid load curve considering demand side response and output of regional wind farm and photovoltaic generation[J]. Power System Technology, 2014, 38(7): 1806-1811.

[24] Li Chunyan, Xu Zhong, Ma Zhiyuan. Optimal time-of-use electricity price model considering customer demand response [J]. Proceedings of the CSU-EPSA, 2015, 27(3): 11-16.

[25] Liu Jidong, Han Xueshan, Han Weiji, et al. Model and algorithm of customers' responsive behavior under time-of-use proce [J]. Power System Technology, 2013, 37(10): 2973-2978.

[26] Dong Kaisong, Ding Yan, Xie Yongtao, et al. Market optimization model for microgrid with demand response [J]. High Voltage Apparatus, 2015, 51(6): 122-126.

[27] Zhang Liying, Meng Lingjia, Wang Zezhong. Photovoltaic power station output power prediction based on the double BP neural network [J]. Electrical Measurement \& Instrumentation, 2015, 52(11): 31-35.

[28] Zhu Yongqiang, Tian Jun. Application of least square support vector machine in photovoltaic power forecasting [J]. Power System Technology, 2011, 35(7): 54-59.

[29] Ding Ming, Xu Ningzhou. A method to forecast short-term output power of photovoltaic generation system based on markovchain [J]. Power System Technology, 2011, 35(1): 152-157.

[30] Jayaweera D, Islam S. Security assessment in active distribution networks with change in weather patterns[C]// International Conference on Probabilistic Methods Applied To Power Systems. IEEE, 2014.

[31] Zhou Chao, Xing Wenyang, Li Yulong. Summarization on load forecasting method of electrical power system [J]. Journal of Power Supply, 2012(6): 32-39. 